Scientific Paper

\title{
Dosimetric comparison of jaw tracking in intensity modulated and volumetric modulated arc radiotherapy for carcinoma of cervix
}

\author{
Karthick Raj MANI ${ }^{1, a}$, Muhtasim Aziz MUNEem ${ }^{1,2}$, Nazneen SULTANA ${ }^{2}$, Tanjina Hossain², \\ Tabinda BASHARAT ${ }^{2}$, Saumen BASU ${ }^{1}$, Ramaa LINGAIAH ${ }^{1}$ \\ ${ }^{I}$ Department of Radiation Oncology, United Hospital, Gulshan, Dhaka, Bangladesh \\ ${ }^{2}$ Department of Biomedical Physics \& Technology, University of Dhaka, Dhaka, Bangladesh \\ ${ }^{a}$ E-mail address: karthickrajmani@gmail.com
}

(received 22 January 2019; revised 3 July 2019; accepted 30 July 2019)

\begin{abstract}
Aim: To study the dosimetric advantages of the jaw tracking technique in intensity-modulated radiotherapy (IMRT) and volumetric modulated arc radiotherapy (VMAT) for carcinoma of cervix patients.

Materials and Methods: We retrospectively selected ten previously treated cervix patients in this study. All the ten patients underwent CT simulation along with immobilization and positional devices. Targets and organ at risks (OARs) were delineated slice by slice for all the patients. All the patients were planned for IMRT and VMAT with intend to deliver $50 \mathrm{~Gy}$ in 25 fractions. All the plans were planned with $6 \mathrm{MV}$ photon beam using millennium-120 multi leaf collimator (MLC) using the TrueBeam linear accelerator. IMRT and VMAT plans were performed with jaw tracking (JT) and with static jaw (SJ) techniques by keeping the same constraints and priorities for the target volumes and critical structures for a particular patient. For standardization, all the plans were normalized to the target mean of the planning target volume. All the plans were accepted with the criteria of bladder mean dose $<40 \mathrm{~Gy}$ and rectum mean dose $<40$ Gy without compromising the target volumes. Target conformity, dose to the critical structures and low dose volumes were recorded and analyzed for IMRT and VMAT plans with and without jaw tracking for all the patients.

Results: The conformity index average of all patients followed by standard deviation $\left(\overline{\mathrm{x}} \pm \sigma_{\overline{\mathrm{x}}}\right)$ for JT-IMRT, SJ-IMRT, JT-VMAT and SJ-VMAT were $1.176 \pm 0.139,1.175 \pm 0.139,1.193 \pm 0.220$ and $1.228 \pm 0.192$ and homogeneity index were $0.089 \pm 0.022,0.085 \pm 0.024,0.102 \pm 0.016$ and $0.101 \pm 0.016$. In low dose volume J,T-IMRT shows a $5.4 \%$ (pvalue < 0.001$)$ overall reduction in volume receiving at least $5 \mathrm{~Gy}\left(\mathrm{~V}_{5}\right)$ compared to SJ-IMRT, whereas $1.2 \%$ reduction was observed in V5 volume in JT-VMAT compared to SJ-VMAT. JT-IMRT showed mean reduction in rectum and bladder of $1.34 \%$ (p-value < 0.001 ) and $1.46 \%$ (p-value < 0.001 ) compared to SJ-IMRT, while only $0.30 \%$ and $0.03 \%$ reduction were observed between JT-VMAT and SJ-VMAT. JT-IMRT plans also showed considerable dose reduction to inthe testine, right femoral head, left femoral head and cauda compared to the SJ-IMRT plans.

Conclusion: Jaw tracking resulted in decreased dose to critical structures in IMRT and VMAT plans. But significant dose reductions were observed for critical structures in the JT-IMRT compared to SJ-IMRT technique. In JT-VMAT plans dose reduction to the critical structures were not significant compared to the JT-IMRT due to relatively lesser monitor units in the VMAT plans.
\end{abstract}

Key words: IMRT; VMAT; radiotherapy; jaw tracking; cervix carcinoma.

\section{Introduction}

In the recent years, the clinical use of intensity-modulated radiation therapy (IMRT) and volumetric modulated arc radiotherapy (VMAT) has drastically increased due to its capability to escalate the dose to the tumor by limiting the doses to the organ at risks (OARs). In sliding window IMRT, the secondary jaws stay in fixed positions whereas the multileaf collimators (MLC) dynamically modulate the intensity of the photon beams according to the fluence pattern. VMAT is an advanced form of IMRT, where the intensity modulation is performed using MLC in a volumetric manner. In the standard static jaw (SJ) IMRT and VMAT delivery, the secondary jaws stay in fixed position and the MLC moves to modulate the intensity pattern of the photon beams to achieve a desired dose distribution. The typical MLC transmission of the Varian Millenium MLC ranges from $1.6 \%$ to $2.5 \%$ for the beam energies from $6 \mathrm{MV}$ to $18 \mathrm{MV}$ photon beams [1]. In 3dimensional conformal radiotherapy (3DCRT) with $15 \mathrm{MV}$ four field box technique the average MU required is $228.9 \pm$ 
3.6 MU for 2 Gy per fraction according to our institutional data, this may vary depends upon the patient thickness and the photon beam energy used, whereas the average MU documented in this study for the static jaw IMRT and VMAT were $1573.6 \pm 206.1$ and $479.6 \pm 124.1$ respectively. Compared to 3DCRT, IMRT monitor units (MU) were approximately 8 fold higher, whereas in VMAT the increase in MU is around 2 to 3 folds. Increased MU in the IMRT and VMAT will result in increased doses to the OARs and normal tissues due to the increase in the MLC transmission, the effect will be predominant in IMRT rather than VMAT. To reduce the MLC transmission effect in IMRT and VMAT, jaw tracking method has been introduced, which brings the secondary jaws close to the open MLC segments. By combining the jaws with MLC, the transmission can be brought down to less than $0.1 \%$ [2].

Varian medical system has introduced the jaw tracking feature in there TrueBeam medical linear accelerator, which reduces the MLC transmission by dynamically tracking all the secondary jaws (Y1, Y2, X1, and X2) close to the MLC aperture during the VMAT and IMRT delivery [2]. The secondary jaws move with a maximum velocity of $2 \mathrm{~cm} / \mathrm{sec}$ to move close to the MLC aperture to minimize MLC transmission.

Eifel et al. [3] described late toxicity in a retrospective study of 1784 patients of FIGO stage IB carcinoma of the cervix treated with conventional radiotherapy between 1960 and 1989. They suffered $7.7 \%$ and $9.3 \% \geq$ grade 3 genitourinary complications at 3 and 5 years respectively. He also concluded that after 5 years the risk continuously increased by $0.34 \%$ per year, resulting in major complications of $14.4 \%$ at 20 years. Intensity-modulated radiation therapy side effects were comparatively lower than conventional radiotherapy; the favorable outcomes described its safety and efficacy of cervical cancer [4]. Clinical use of IMRT and VMAT in carcinoma of the cervix has considerably increased in the last decade due to its unique features of confirming the high dose volumes on the target volumes and sparing the OARs. The low dose volumes were always major concerns in the young adult and pediatric patients, which has a well-established relationship with the secondary malignancies [5]. IMRT and VMAT techniques with jaw tracking will definitely able to reduce the low dose volumes and spare OAR's.

The dosimetric influence of jaw tracking in IMRT and VMAT for head and neck cancer has been investigated and published in our previous study [1]. In this study, we would like to investigate the dosimetric influences of jaw tracking IMRT and VMAT compared to the static jaw IMRT and VMAT in the patients of carcinoma of the cervix, as an extension of our previous study.

\section{Materials and Methods}

We retrospectively selected ten previously treated patients diagnosed with carcinoma of the cervix. All the patients were planned for IMRT and VMAT with static jaw and jaw tracking techniques. We intended to deliver a total dose of $50 \mathrm{~Gy}$ in 25 fractions with a daily dose of $2 \mathrm{~Gy}$ per fraction. The patient demographic data were listed in Table 1.

Table 1. Patient demographic data

\begin{tabular}{lccc}
\hline \hline Patients & Age (Year) & $\begin{array}{c}\text { Histopathological } \\
\text { Report (HPR) }\end{array}$ & Stage \\
\hline Patient 1 & 47 & Adenocarcinoma & III (Post Op.) \\
Patient 2 & 45 & Squamous Cell Carcinoma & IB \\
Patient 3 & 50 & Squamous Cell Carcinoma & IIIB \\
Patient 4 & 48 & Squamous Cell Carcinoma & IB \\
Patient 5 & 40 & Squamous Cell Carcinoma & IB \\
Patient 6 & 53 & Squamous Cell Carcinoma & IB2 \\
Patient 7 & 42 & Adenocarcinoma & IIA \\
Patient 8 & 46 & Squamous Cell Carcinoma & IIB \\
Patient 9 & 46 & Endometrioid Adenocarcinoma & IA \\
Patient 10 & 57 & Squamous Cell Carcinoma & CIN3 \\
\hline \hline
\end{tabular}

\section{CT simulation and delineation}

All the ten patients were immobilized with vac-lock (CIVCO, Orange City, IA, USA) indexed to the couch. Contrastenhanced axial slices with a slice thickness of $2.5 \mathrm{~mm}$ were obtained for all the patients using GE Discovery 60016 slice PET/CT scanner (GE Healthcare, Waukesha, WI, USA). Dicom images of the patients were transferred to the ARIA (Varian Medical Systems, Palo Alto, California, USA) oncology record and verification software using the file transfer protocol. The received images were imported into the ARIA and the demographic data of the patients were assigned accordingly. Body structure contours were auto segmented in the treatment planning system. The contouring of the gross tumor volume (GTV), the clinical target volume (CTV) (includes the subclinical diseases and nodal stations) and OARs (bladder, rectum, right femoral head, left femoral head, intestine, and cauda) were delineated by the same radiation oncologist to avoid inter-observer variability. Illustration of organ at risks and target volumes delineation were listed in Figure 1.

\section{Treatment Planning}

Ten patients were planned with jaw tracking and static jaw IMRT and VMAT with a total dose prescription of $50 \mathrm{~Gy}$ in 25 fractions with a daily dose of 2 Gy per fraction. All the patients were planned with TrueBeam linear accelerator in Eclipse treatment planning system using $6 \mathrm{MV}$ flattened beam for all the plans along with the millennium-120 MLC for the beam modulation. The $6 \mathrm{MV}$ MLC transmission for the millennium120 MLC and the jaw transmission used in this study were $1.90 \%$ and $0.41 \%$ respectively. Seven fields (gantry angles $0^{\circ}$, $51^{\circ}, 102^{\circ}, 153^{\circ}, 204^{\circ}, 255^{\circ}$, and $306^{\circ}$ ) were used for IMRT plans with jaw tracking and static jaw, whereas two complete arcs with $\pm 30^{\circ}$ collimator angle were used for VMAT plans. The beam's eye view of the jaw tracking and static jaw IMRT and VMAT segments were illustrated in Figure 2. 

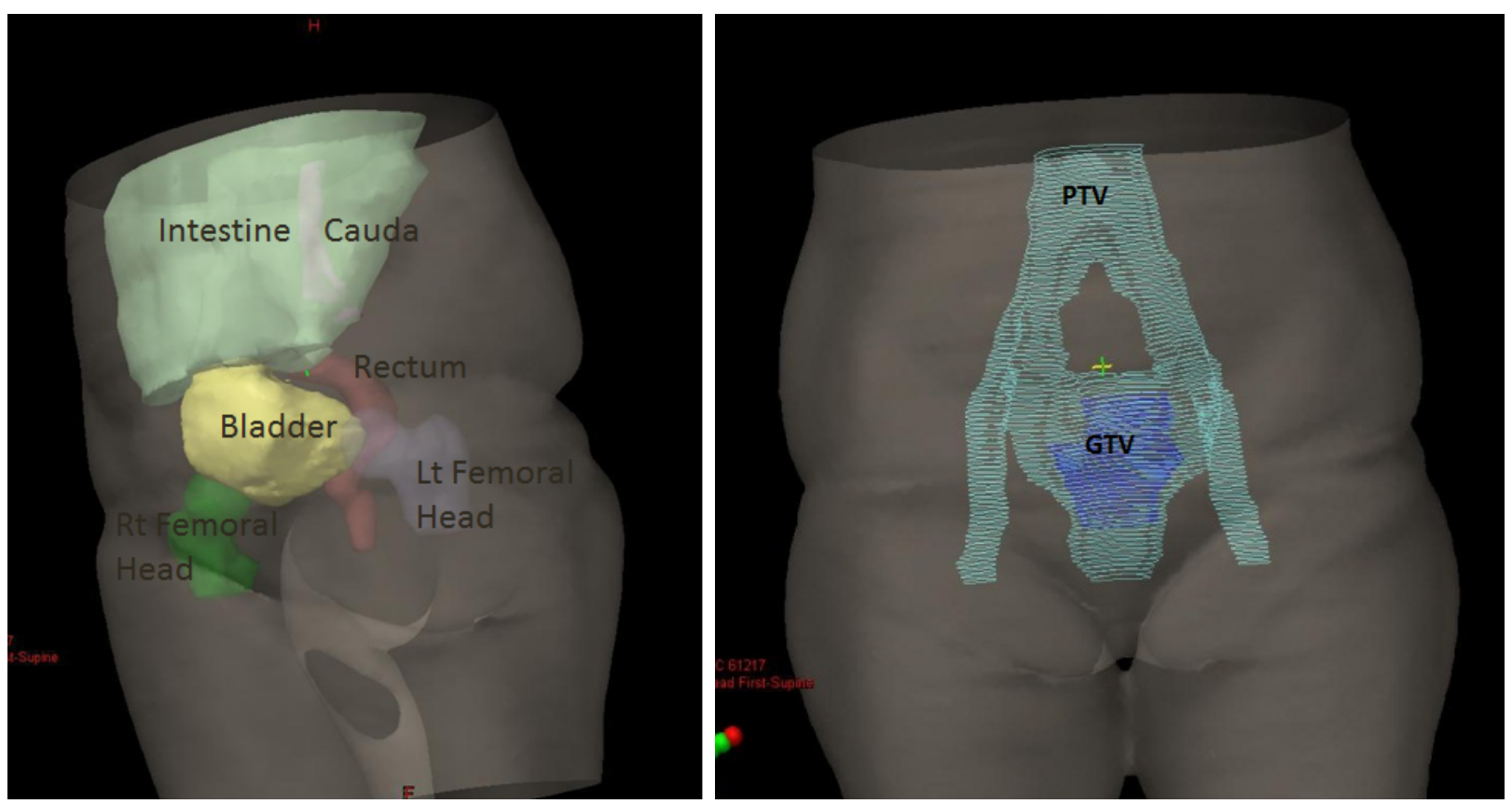

Figure 1. Illustration of organ at risks and target volumes delineation
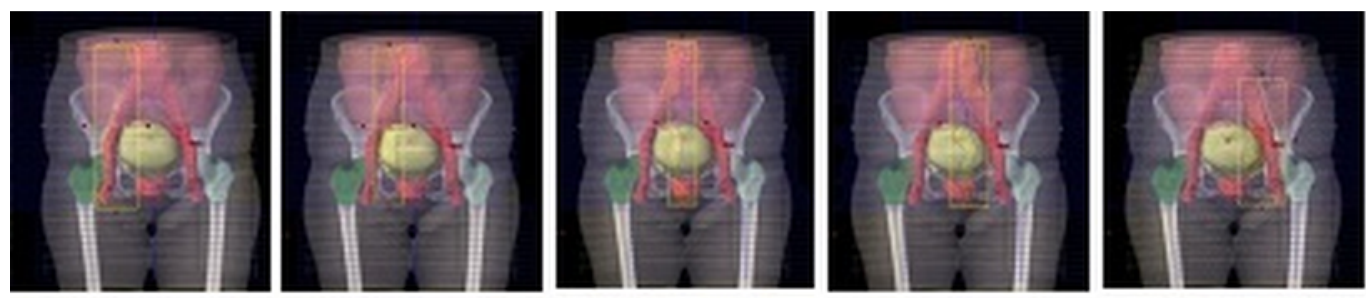

a
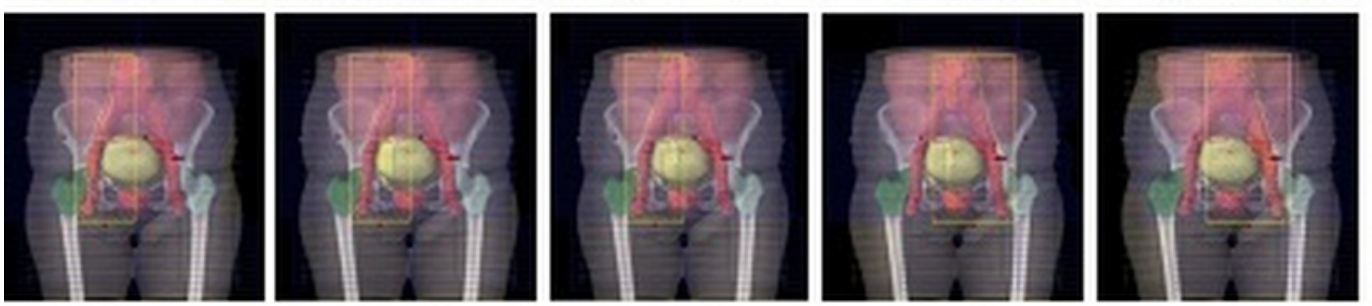

b
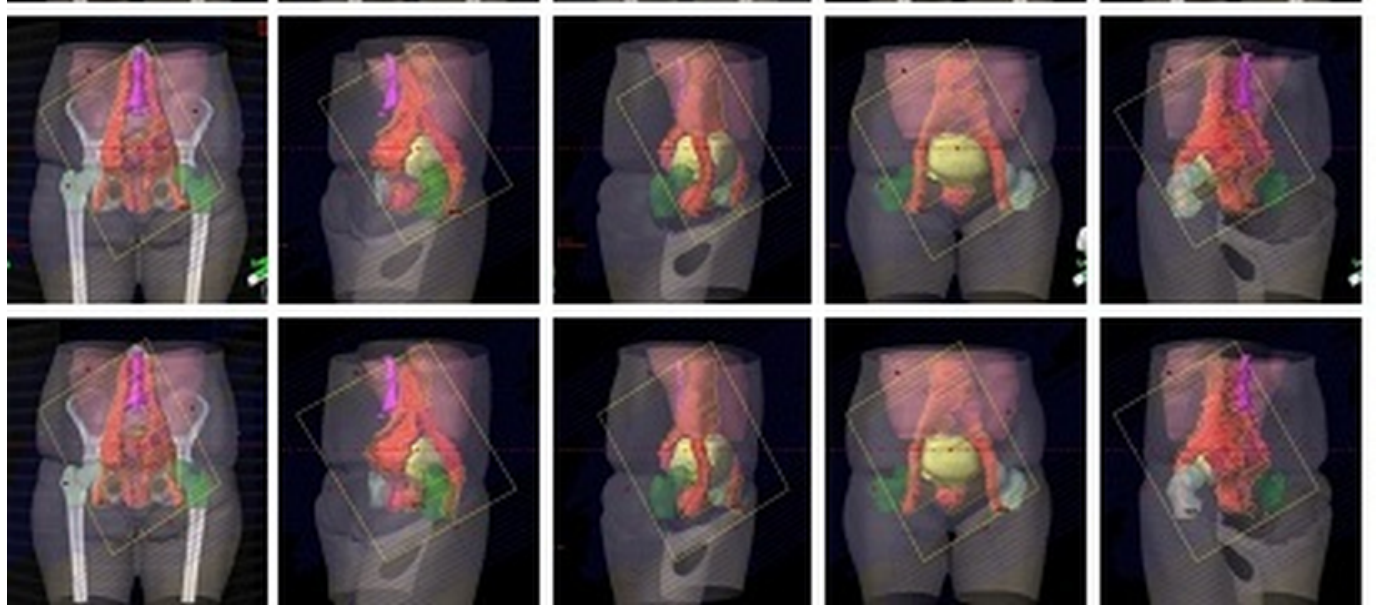

$\mathrm{C}$

d

Figure 2. (a) JT-IMRT field segments (b) SJ-IMRT field segments (c) JT-VMAT arc segments (d) SJ-VMAT arc segments. 
Dose-volume optimizer ver. 11.0.31 was used for IMRT optimization along with smart leaf motion calculator to convert the optimal fluences to actual fluences. Progressive resolution optimizer ver. 11.0.31 was used for VMAT optimization. Analytical anisotropic algorithm ver. 11.0.31 dose calculation algorithm was used with $2.5 \mathrm{~mm}$ grid resolution for both IMRT and VMAT plans.

For a particular patient, IMRT and VMAT plans with jaw tracking and static jaw was optimized with the same dose constraints and priorities by enabling and disabling jaw tracking during the optimization. For standardization, all the plans were normalized to the target mean of the PTV.

\section{Plan quality assessment}

Dose conformity and homogeneity are independent specifications of the quality of the absorbed dose distribution. Dose conformity characterizes the degree to which the high dose region conforms to the target volume whereas dose homogeneity characterizes the uniformity of the absorbed dose within the target volume. The plan quality of the jaw tracking and static jaw IMRT and VMAT were evaluated using the homogeneity index and conformity index. The homogeneity index defined by the International Commission of Radiological Units (ICRU) report no 83 published [6] in 2010 was used to compare the plan homogeneity. The conformity index recommended by the Radiation therapy oncology group (RTOG) in 1993 described in Equation 2 was used to compare the plan conformity.

\section{Homogeneity Index (HI)}

ICRU-83, defines the homogeneity index as below

Homogeneity Index $(H I)=\frac{D_{2 \%}-D_{98 \%}}{D_{50 \%}}$

Where,

$\mathrm{D}_{2 \%}, \mathrm{D}_{98 \%}$ and $\mathrm{D}_{50 \%}$ are the doses received by the volume $2 \%$, $98 \%$ and $50 \%$ respectively.

$\mathrm{HI}=0.000$ (zero) is ideal value .

Conformity Index (CI)

RTOG has recommended the conformity index as a ratio of the volume of the PTV covered by the reference dose to the total volume of the PTV.

Conformity Index $\left(C I_{R T O G}\right)=\frac{V_{R I}}{T V}$

Where,

$\mathrm{V}_{\mathrm{RI}}=$ volume of PTV covered by the reference dose, TV is the volume of PTV.

Target conformity and homogeneity were calculated using the above Equation $\mathbf{1}$ and $\mathbf{2}$ for all the jaw tracking and static jaw IMRT/VMAT plans to evaluate the plan quality. The dose to the OARs and normal tissue low dose volumes $\left(\mathrm{V}_{5}, \mathrm{~V}_{10}, \mathrm{~V}_{20}\right.$, and $V_{30}$, where $V_{n}$ is the percentage volume receiving at least ' $n$ ' Gy) were recorded and analyzed for IMRT and VMAT plans with and without jaw tracking for all the patients. $\mathrm{V}_{5}$, $\mathrm{V}_{10}, \mathrm{~V}_{20}$, and $\mathrm{V}_{30}$ were selected to compare the dosimetric differences between the jaw tracking and static jaw plans.

\section{Patient-specific QA}

Patient-specific QA for all the ten patients planned with JTIMRT, SJ-IMRT, JT-VMAT, and SJ-VMAT (total 40 plans), were performed using Varian portal dosimetry. The treatment planning system (TPS) creates a reference fluence of the individual treatment plans of IMRT and VMAT using the portal dose image prediction (PDIP) algorithm. The aS1000 amorphous silicon portal imager with image acquisition system 3 attached to the exact arm, were used for the acquisition the delivered plan images. The gamma analysis with a $3 \%$ dose and $3 \mathrm{~mm}$ distance to agreement (DTA) criteria were used to compare the acquired images with the TPS predicated fluence. The region of interest for portal dosimetry analysis includes MLC complete irradiated area outline (CIAO) with an additional $1 \mathrm{~cm}$ margin. The gamma analysis pass criteria were set as $95 \%$ of the pixels should pass the $3 \%$ dose and $3 \mathrm{~mm}$ DTA between the acquired images with the TPS predicated fluence.

\section{Statistical Data Analysis}

All the statistical data presented in this work, are as a mean of all the data followed by the standard deviation $\left(\bar{X} \pm \sigma_{\bar{X}}\right)$. The paired sample's T-test between the jaw tracking and static jaw techniques were performed using the Microsoft Word/Excel version 2010 with $\mathrm{p}<0.05$ considered as significant.

\section{Results}

\section{Conformity Index and Homogeneity Index}

The plan quality of the IMRT and VMAT plans were evaluated using the conformity index (CI) and the homogeneity index (HI). The obtained conformity and homogeneity index for the static jaw and jaw tracking techniques were tabulated in Table 2. The results listed in Table 2 are the average of all the patients analyzed in this study. Mean conformity index followed by the standard deviation for JT-IMRT, SJ-IMRT, JTVMAT, and SJ-VMAT techniques were $1.176 \pm 0.139,1.175 \pm$ $0.139,1.193 \pm 0.220$ and $1.228 \pm 0.192$ respectively. The mean homogeneity index for JT-IMRT, SJ-IMRT, JT-VMAT and SJVMAT were $0.089 \pm 0.022,0.085 \pm 0.024,0.102 \pm 0.016$ and $0.101 \pm 0.016$ respectively. The CI and HI parameters with and without jaw tracking IMRT / VMAT plans were comparable. The homogeneity and the conformity index parameters for all the patients were listed in Table 2. 
Table 2. Homogeneity and Conformity Index

\begin{tabular}{|c|c|c|c|c|}
\hline Parameter & JT-IMRT & SJ-IMRT & JT-VMAT & SJ-VMAT \\
\hline $\mathrm{D}_{2} \%(\mathrm{~Gy})$ & $51.957 \pm 0.511$ & $51.944 \pm 0.524$ & $52.120 \pm 0.237$ & $52.093 \pm 0.233$ \\
\hline $\mathbf{D}_{\mathbf{5 0}} \%(\mathbf{G y})$ & $50.060 \pm 0.050$ & $50.251 \pm 0.616$ & $50.121 \pm 0.069$ & $50.119 \pm 0.071$ \\
\hline $\mathrm{D}_{98} \%(\mathrm{~Gy})$ & $47.477 \pm 0.625$ & $47.651 \pm 0.751$ & $47.018 \pm 0.591$ & $47.050 \pm 0.591$ \\
\hline $95 \%$ isodose volume (cc) & $1233.947 \pm 325.200$ & $1229.530 \pm 322.959$ & $1237.88 \pm 319.77$ & $1243.82 \pm 324.39$ \\
\hline PTV volume (cc) & \multicolumn{4}{|c|}{$1035.606 \pm 308.383$} \\
\hline CI $\mathbf{I}_{(\mathbf{R T O G})}$ & $1.176 \pm 0.139$ & $1.175 \pm 0.139$ & $1.193 \pm 0.220$ & $1.228 \pm 0.192$ \\
\hline HI & $0.089 \pm 0.022$ & $0.085 \pm 0.024$ & $0.102 \pm 0.016$ & $0.101 \pm 0.016$ \\
\hline
\end{tabular}

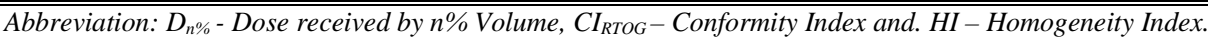

\section{Low dose volumes (Body)}

Comparison between the low dose volume $\left(\mathrm{V}_{5}, \mathrm{~V}_{10}, \mathrm{~V}_{20}\right.$, and $\mathrm{V}_{30}$ ) for JT and SJ techniques for IMRT and VMAT were listed in Table 3 and Table 4. In the IMRT plans, the JT technique resulted in significant doses reduction compared to SJ technique. The percentage dose difference of the JT-IMRT plans were $5.396 \%, 3.577 \%, 7.742 \%$ and $4.407 \%$ for the $\mathrm{V}_{5}$, $\mathrm{V}_{10}, \mathrm{~V}_{20}$, and $\mathrm{V}_{30}$ respectively. The mean reduction dose to the whole body was $4.219 \%$ in JT-IMRT compared to SJ-IMRT and it is statistically significant $(p=0.023)$. The DVH comparison between JT-IMRT plans and SJ-IMRT plans for the low dose volume has been shown in Figure 3.

The JT-VMAT plans displayed dose reduction in the $\mathrm{V}_{5}, \mathrm{~V}_{10}$, $\mathrm{V}_{20}$ and $\mathrm{V}_{30}$ volumes by $0.926 \%, 1.188 \%, 1.410 \%$, and $1.426 \%$ and mean doses reduction of the whole body by $1.197 \%$ compared to the SJ-VMAT. Statistically significant dose reductions were observed in the $\mathrm{V}_{5}, \mathrm{~V}_{10}, \mathrm{~V}_{20}, \mathrm{~V}_{30}$ and mean dose of the body ( $\mathrm{p}<0.005)$. The DVH comparison of low dose volumes for a patient between JT-VMAT and SJ-VMAT is shown in Figure 3.

\section{Bladder}

Statistical analysis of the bladder volume $\mathrm{V}_{5}, \mathrm{~V}_{10}, \mathrm{~V}_{20}$ and $\mathrm{V}_{30}$ between JT and SJ techniques for IMRT were listed in Table 3 and VMAT in Table 4. In the IMRT plans, the JT technique resulted in significant dose reduction compared to the SJ technique. The percentage dose difference of the JT-IMRT plans was $0.034 \%, 1.511 \%$ and $3.220 \%$ for $\mathrm{V}_{10}, \mathrm{~V}_{20}$, and $\mathrm{V}_{30}$ respectively. $\mathrm{V}_{5}$ for both JT-IMRT and SJ-IMRT plans were similar. The mean reduction dose to the bladder was $1.459 \%$ in JT-IMRT compared to SJ-IMRT and it is statistically significant $(\mathrm{p}<0.001)$. Dose-volume histogram comparison of organ at risk and PTV for JT-IMRT and SJ-IMRT were illustrated in Figure 4.

The JT-VMAT plans resulted in dose reduction in $\mathrm{V}_{20}$ and $\mathrm{V}_{30}$ volumes by $0.086 \%$ and $0.201 \%$. Mean dose to the bladder was reduced by $0.469 \%$ compared to the SJ-VMAT. Dose reductions were observed in $\mathrm{V}_{20}$ and $\mathrm{V}_{30}$ but they were not statistically significant. $\mathrm{V}_{5}$ and $\mathrm{V}_{10}$ in both JT-IMRT and SJIMRT plans were similar. Dose-volume histogram comparison of organ at risk and PTV for JT-VMAT and SJ-VMAT were illustrated in Figure 5.

\section{Rectum}

Comparison of the rectum volumes $\left(\mathrm{V}_{5}, \mathrm{~V}_{10}, \mathrm{~V}_{20}\right.$, and $\left.\mathrm{V}_{30}\right)$ between JT and SJ techniques for IMRT were listed in Table 3 and VMAT in Table 4. In the IMRT plans, the JT technique resulted in significant doses reduction compared to SJ technique. The percentage dose difference of the JT-IMRT plans were $0.044 \%, 1.207 \%$ and $3.250 \%$ for $\mathrm{V}_{10}, \mathrm{~V}_{20}$, and $\mathrm{V}_{30}$ respectively. $\mathrm{V}_{5}$ for both JT-IMRT and SJ-IMRT plans were similar. The mean reduction dose to the rectum was $1.348 \%$ in JT-IMRT compared to SJ-IMRT and it is statistically significant $(\mathrm{p}<0.001)$.

The JT-VMAT plans displayed dose reduction in $\mathrm{V}_{10}, \mathrm{~V}_{20}$, and $\mathrm{V}_{30}$ volumes by $0.060 \%, 0.154 \%$, and $0.026 \%$. Mean doss to the bladder was reduced by $0.298 \%$ compared to the SJVMAT. Dose reductions were observed in $\mathrm{V}_{10}, \mathrm{~V}_{20}$, and $\mathrm{V}_{30}$ but they were not statistically significant. $\mathrm{V}_{5}$ in both JT-IMRT and SJ-IMRT plans were similar.

\section{Right femoral head}

Dose reductions in the right femoral head V5, V10, V20, and V30 between JT and SJ techniques for IMRT were listed in Table 3 and VMAT in Table 4. In the IMRT plans, the JT technique resulted in significant doses reduction compared to SJ technique. The percentage dose difference of the JT-IMRT plans was $0.574 \%, 2.942 \%, 13.670 \%$ and $4.320 \%$ for $\mathrm{V}_{5}, \mathrm{~V}_{10}$, $\mathrm{V}_{20}$, and $\mathrm{V}_{30}$ respectively. The mean reduction dose to the right femoral head was $4.215 \%$ in JT-IMRT compared to SJ-IMRT and it is statistically significant $(\mathrm{p}<0.001)$.

The JT-VMAT plans displayed dose reduction in $\mathrm{V}_{5}, \mathrm{~V}_{10}$, $\mathrm{V}_{20}$, and $\mathrm{V}_{30}$ volumes by $1.162 \%, 1.525 \%, 3.214 \%$, and $0.246 \%$. Mean dose to the right femoral head was reduced by $1.877 \%$ compared to the SJ-VMAT. Dose reductions were observed in $\mathrm{V}_{5}, \mathrm{~V}_{10}, \mathrm{~V}_{20}$, and $\mathrm{V}_{30}$ but they were not statistically significant.

\section{Left femoral head}

Femoral head volumes $\left(\mathrm{V}_{5}, \mathrm{~V}_{10}, \mathrm{~V}_{20}\right.$, and $\left.\mathrm{V}_{30}\right)$ along with their mean dose in JT and SJ techniques for IMRT and VMAT were listed in Table 3 and Table 4. In the IMRT plans, the JT technique resulted in significant doses reduction compared to SJ technique. The percentage dose difference of the JT-IMRT plans was $0.316 \%, 1.399 \%, 12.007 \%$ and $3.439 \%$ for $\mathrm{V}_{5}, \mathrm{~V}_{10}$, 
$\mathrm{V}_{20}$, and $\mathrm{V}_{30}$ respectively. The mean reduction dose to the left femoral head was $2.886 \%$ in JT-IMRT compared to SJ-IMRT and it is statistically significant $(\mathrm{p}<0.001)$.

The JT-VMAT plans displayed dose reduction in $\mathrm{V}_{5}, \mathrm{~V}_{10}$, $\mathrm{V}_{20}$ and $\mathrm{V}_{30}$ volumes by $0.082 \%, 3.902 \%, 0.219 \%$, and $0.464 \%$. Mean doses to the Left femoral head was reduced by $0.949 \%$ compared to the SJ-VMAT. Though dose reductions were observed in $\mathrm{V}_{5}, \mathrm{~V}_{10}, \mathrm{~V}_{20}$, and $\mathrm{V}_{30}$, the differences were not statistically significant.

\section{Intestine}

Comparison between the intestine volumes $\mathrm{V}_{5}, \mathrm{~V}_{10}, \mathrm{~V}_{20}$ and $\mathrm{V}_{30}$ between $\mathrm{JT}$ and $\mathrm{SJ}$ techniques for IMRT and VMAT were listed in Table 3 and Table 4. In the IMRT plans, the JT technique resulted in significant doses reduction compared to SJ technique. The percentage dose difference of the JT-IMRT plans were $1.190 \%, 2.468 \%, 6.149 \%$ and $6.571 \%$ for $\mathrm{V}_{5}, \mathrm{~V}_{10}$, $\mathrm{V}_{20}$, and $\mathrm{V}_{30}$ respectively. The mean reduction dose to the intestine was $3.198 \%$ in JT-IMRT compared to SJ-IMRT and it is statistically significant ( $\mathrm{p}<0.001)$.

The JT-VMAT plans displayed dose reduction in $\mathrm{V}_{5}, \mathrm{~V}_{10}$, $\mathrm{V}_{20}$ and $\mathrm{V}_{30}$ volumes by $0.610 \%, 0.124 \%, 1.113 \%$, and $0.578 \%$. Mean dose to the intestine was reduced by $0.449 \%$ compared to the SJ-VMAT. Dose reductions were observed in $\mathrm{V}_{5}, \mathrm{~V}_{10}, \mathrm{~V}_{20}$, and $\mathrm{V}_{30}$ but they were not statistically significant.

\section{Cauda}

Statistical analysis of the cauda volumes $\left(\mathrm{V}_{5}, \mathrm{~V}_{10}, \mathrm{~V}_{20}\right.$, and $\left.\mathrm{V}_{30}\right)$ between JT and SJ techniques for IMRT and VMAT were listed in Table 3 and Table 4. In the IMRT plans, the JT

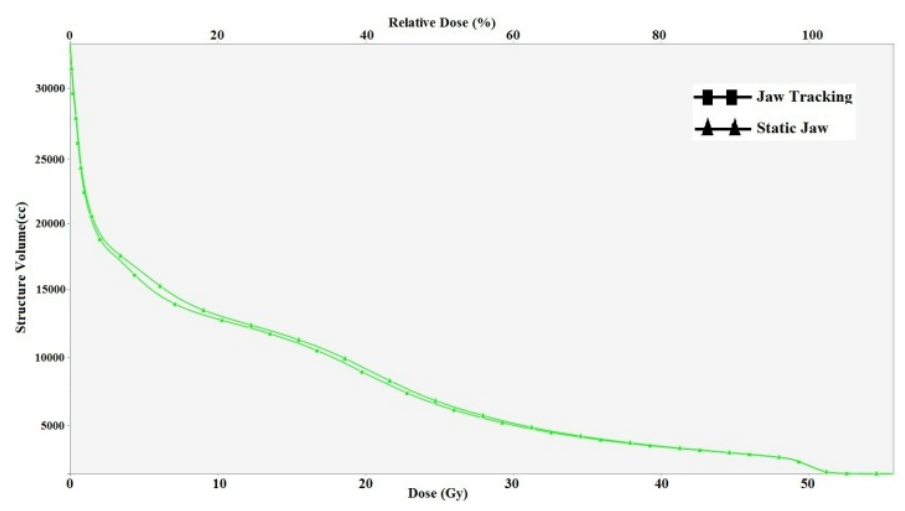

(a) technique resulted in significant doses reduction compared to SJ technique. The percentage dose difference of the JT-IMRT plans was $0.946 \%, 0.866 \%, 0.856 \%$ and $8.423 \%$ for the $V_{5}$, $\mathrm{V}_{10}, \mathrm{~V}_{20}$, and $\mathrm{V}_{30}$ respectively. The mean reduction dose to the cauda was $0.258 \%$ in JT-IMRT compared to SJ-IMRT and it is statistically significant $(\mathrm{p}=0.043)$. Dose reduction in maximum point dose was $0.375 \%$.

The JT-VMAT plans displayed dose reduction in $\mathrm{V}_{5}, \mathrm{~V}_{10}$, $\mathrm{V}_{20}$, and $\mathrm{V}_{30}$ volumes by $1.896 \%, 0.462 \%, 0.423 \%$, and $1.756 \%$. Mean doses to the intestine was reduced by $0.268 \%$ compared to the SJ-VMAT. The maximum point dose was reduced by $0.179 \%$ Dose reductions were observed in $\mathrm{V}_{5}, \mathrm{~V}_{10}$, $\mathrm{V}_{20}$, and $\mathrm{V}_{30}$ but the differences were not statistically significant.

\section{Patient-specific QA}

The patient-specific quality assurance using portal dosimetry for all JT-IMRT, SJ-IMRT, JT-VMAT, and SJ-VMAT were performed gamma analysis with $3 \%$ dose and $3 \mathrm{~mm}$ DTA criteria. The mean \pm standard deviation of the percentage of pixels passed using gamma evaluation method for JT-IMRT, SJ-IMRT, JT-VMAT, and SJ-VMAT were $97.798 \pm 1.206$, $97.3296 \pm 1.341,97.193 \pm 1.289$ and $97.832 \pm 1.325$. These QA results provide us the confidence that the TPS predicted and the delivered fluences of all the plans with jaw tracking and static jaw in IMRT/VMAT plans were well within the tolerances and eligible for the treatment delivery.

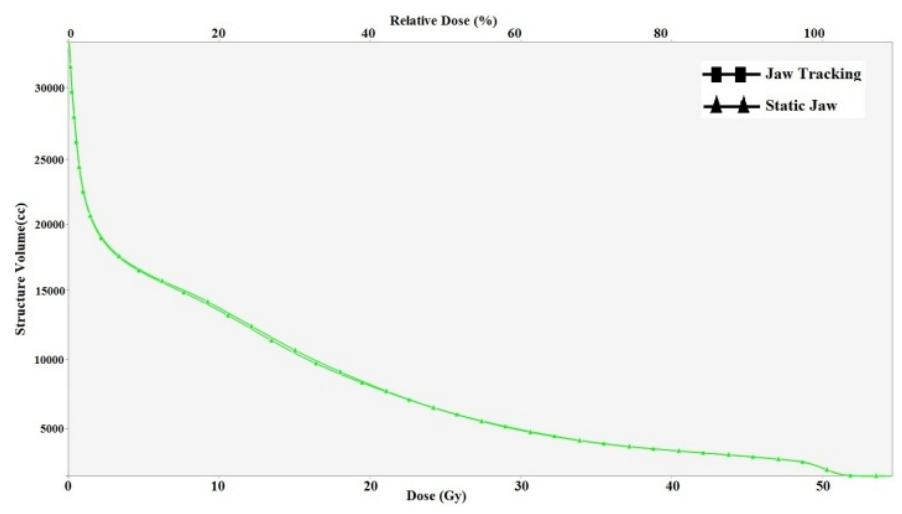

(b)

Figure 3. (a) Dose-volume histogram comparison of the body structure between JT-IMRT and SJ-IMRT and (b) Dose-volume histogram comparison of the body structure between JT-VMAT and SJ-VMAT 


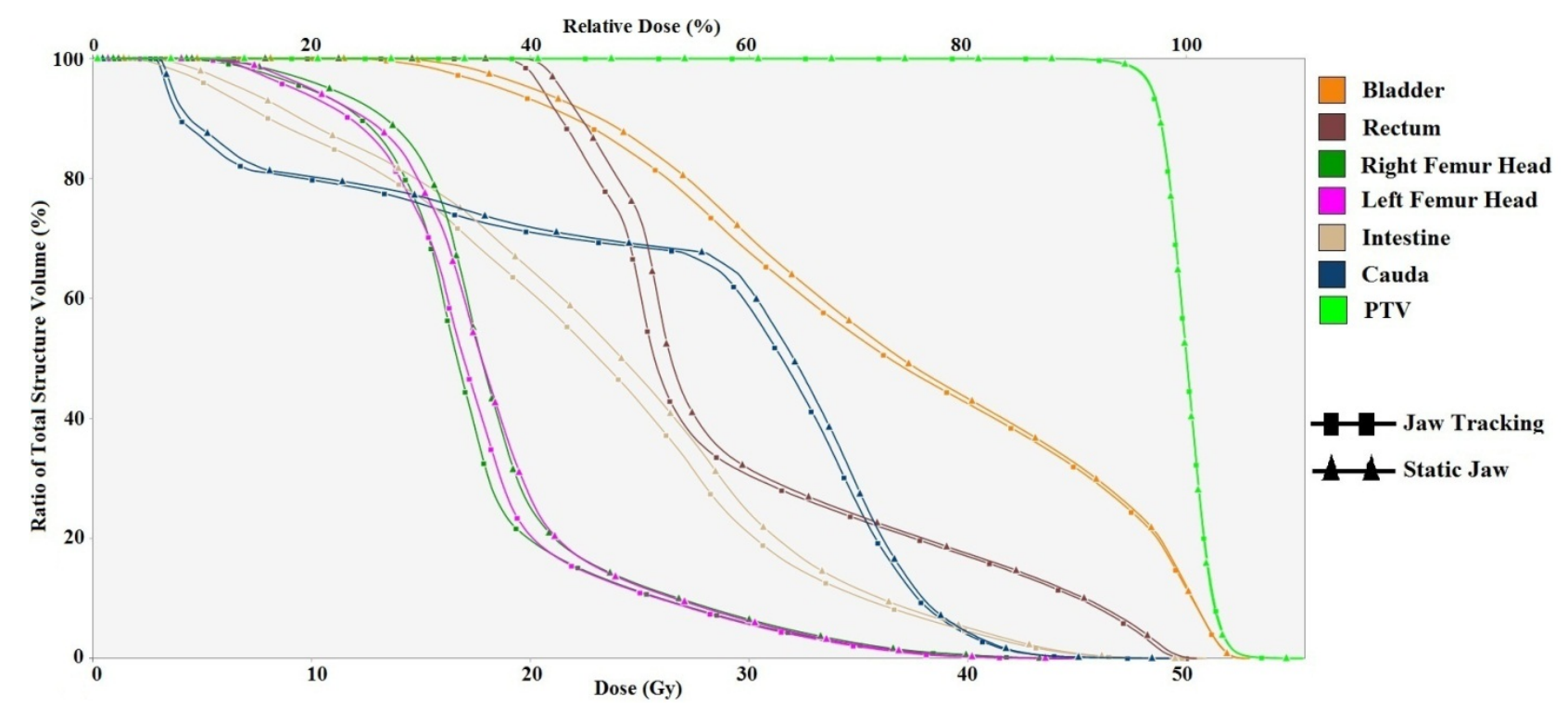

Figure 4. Dose volume histogram comparison of organ at risk and PTV for JT-IMRT and SJ-IMRT

Table 3. Comparison between Jaw Tracking and Static Jaw IMRT

\begin{tabular}{|c|c|c|c|c|c|}
\hline $\begin{array}{c}\text { Organ Volume (cc) } \\
(\overline{\mathbf{x}} \pm \sigma \mathbf{x})\end{array}$ & Parameter & $\begin{array}{c}\text { JT-IMRT } \\
(\overline{\mathbf{x}} \pm \sigma \mathbf{x}) \\
\end{array}$ & $\begin{array}{c}\text { SJ-IMRT } \\
(\overline{\mathbf{x}} \pm \sigma \mathbf{x}) \\
\end{array}$ & Difference (\%) & p-Value \\
\hline Body & V5 (\%) & $49.069 \pm 7.588$ & $51.717 \pm 7.687$ & 5.396 & 0.012 \\
\hline \multirow{4}{*}{$28777.915 \pm 7364.678$} & $\mathrm{~V} 10(\%)$ & $40.869 \pm 6.401$ & $42.333 \pm 6.321$ & 3.577 & 0.038 \\
\hline & $\mathrm{V} 20(\%)$ & $26.710 \pm 3.718$ & $28.778 \pm 4.249$ & 7.742 & 0.004 \\
\hline & V30 (\%) & $13.955 \pm 2.320$ & $14.570 \pm 2.346$ & 4.407 & 0.005 \\
\hline & Mean (Gy) & $12.288 \pm 1.684$ & $12.807 \pm 1.667$ & 4.219 & 0.023 \\
\hline Bladder & V5 (\%) & $100.000 \pm 0.000$ & $100.000 \pm 0.000$ & 0.000 & - \\
\hline \multirow{4}{*}{$313.328 \pm 92.421$} & V10 (\%) & $99.966 \pm 0.108$ & $100.000 \pm 0.000$ & 0.034 & 0.343 \\
\hline & $\mathrm{V} 20(\%)$ & $91.779 \pm 13.193$ & $93.166 \pm 12.191$ & 1.511 & 0.011 \\
\hline & $\mathrm{V} 30(\%)$ & $69.713 \pm 15.743$ & $71.958 \pm 15.746$ & 3.220 & $<0.001$ \\
\hline & Mean(Gy) & $35.381 \pm 3.439$ & $35.897 \pm 3.254$ & 1.459 & $<0.001$ \\
\hline Rectum & V5 (\%) & $100.000 \pm 0.000$ & $100.000 \pm 0.000$ & 0.000 & - \\
\hline \multirow{4}{*}{$69.214 \pm 31.475$} & V10 (\%) & $99.821 \pm 0.566$ & $99.865 \pm 0.427$ & 0.044 & 0.343 \\
\hline & $\mathrm{V} 20(\%)$ & $96.337 \pm 7.711$ & $97.500 \pm 5.431$ & 1.207 & 0.153 \\
\hline & $\mathrm{V} 30(\%)$ & $73.589 \pm 25.136$ & $75.981 \pm 25.025$ & 3.250 & 0.005 \\
\hline & Mean(Gy) & $35.798 \pm 5.094$ & $36.281 \pm 4.849$ & 1.348 & $<0.001$ \\
\hline Right Femur Head & V5 (\%) & $98.970 \pm 2.144$ & $99.538 \pm 0.934$ & 0.574 & 0.174 \\
\hline \multirow{4}{*}{$83.864 \pm 27.033$} & V10 (\%) & $89.617 \pm 8.472$ & $92.253 \pm 6.988$ & 2.942 & 0.008 \\
\hline & $\mathrm{V} 20(\%)$ & $30.684 \pm 13.086$ & $34.879 \pm 12.507$ & 13.670 & $<0.001$ \\
\hline & V30 (\%) & $12.084 \pm 6.807$ & $12.606 \pm 6.804$ & 4.320 & $<0.001$ \\
\hline & Mean(Gy) & $19.031 \pm 2.566$ & $19.833 \pm 2.317$ & 4.215 & $<0.001$ \\
\hline Left Femur Head & V5 (\%) & $99.015 \pm 2.158$ & $99.328 \pm 1.586$ & 0.316 & 0.131 \\
\hline \multirow{4}{*}{$83.329 \pm 25.280$} & V10 (\%) & $93.173 \pm 5.799$ & $94.477 \pm 5.558$ & 1.399 & 0.005 \\
\hline & $\mathrm{V} 20(\%)$ & $38.964 \pm 20.057$ & $43.642 \pm 20.203$ & 12.007 & $<0.001$ \\
\hline & V30 (\%) & $15.441 \pm 10.698$ & $15.972 \pm 10.882$ & 3.439 & $<0.001$ \\
\hline & Mean(Gy) & $20.566 \pm 3.341$ & $21.159 \pm 3.218$ & 2.886 & $<0.001$ \\
\hline Intestine & V5 (\%) & $90.584 \pm 14.269$ & $91.626 \pm 14.112$ & 1.190 & 0.003 \\
\hline \multirow{4}{*}{$1098.571 \pm 417.814$} & $\mathrm{~V} 10(\%)$ & $81.047 \pm 15.553$ & $83.047 \pm 15.187$ & 2.468 & 0.001 \\
\hline & $\mathrm{V} 20(\%)$ & $59.933 \pm 18.985$ & $63.618 \pm 17.944$ & 6.149 & 0.001 \\
\hline & V30 (\%) & $28.637 \pm 15.488$ & $30.519 \pm 15.897$ & 6.572 & $<0.001$ \\
\hline & Mean(Gy) & $22.678 \pm 5.091$ & $23.403 \pm 5.007$ & 3.198 & $<0.001$ \\
\hline \multirow[t]{3}{*}{ Cauda } & V5 (\%) & $84.017 \pm 13.446$ & $84.812 \pm 13.087$ & 0.946 & 0.002 \\
\hline & $\mathrm{V} 10(\%)$ & $78.758 \pm 13.894$ & $79.440 \pm 13.883$ & 0.866 & $<0.001$ \\
\hline & $\mathrm{V} 20(\%)$ & $72.341 \pm 14.517$ & $72.960 \pm 14.540$ & 0.856 & $<0.001$ \\
\hline \multirow[t]{3}{*}{$23.464 \pm 9.838$} & V30 (\%) & $56.376 \pm 19.148$ & $61.125 \pm 18.497$ & 8.423 & 0.004 \\
\hline & Mean(Gy) & $26.464 \pm 4.976$ & $26.396 \pm 5.087$ & 0.258 & 0.911 \\
\hline & $\operatorname{Max}(\mathrm{Gy})$ & $44.538 \pm 4.563$ & $44.705 \pm 4.398$ & 0.375 & 0.043 \\
\hline
\end{tabular}

Abbreviation: $\left(\bar{x} \pm \sigma_{\bar{x}}\right)$ is Mean \pm Standard deviation, $V_{n}(\%)$ is the percentage volume receiving ' $n$ ' Gy, JT- jaw tracking, SJ- static jaw 


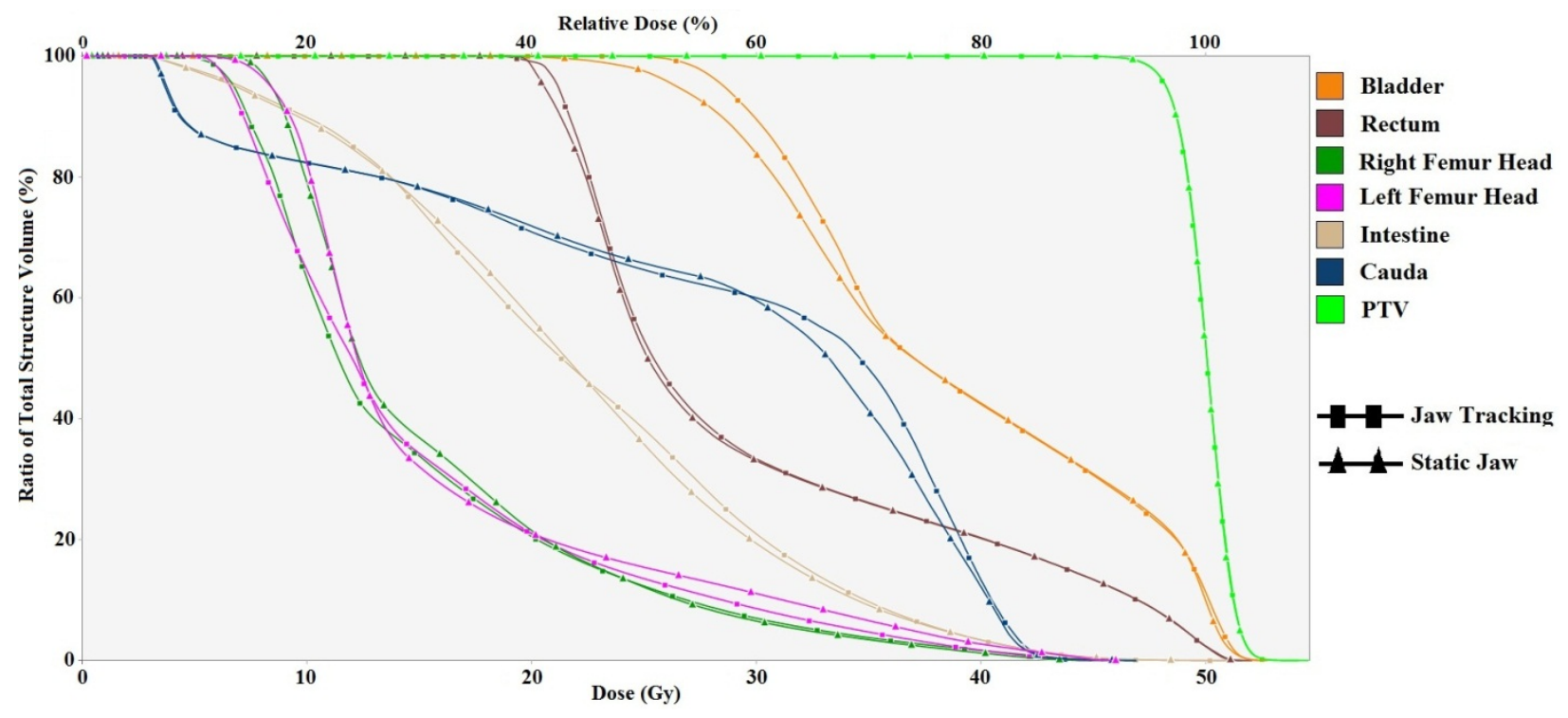

Figure 5. Dose-volume histogram comparison of organ at risk and PTV for JT-VMAT and SJ-VMAT

Table 4. Comparison between Jaw Tracking and Static Jaw VMAT

\begin{tabular}{|c|c|c|c|c|c|}
\hline $\begin{array}{c}\text { Organ Volume (cc) } \\
(\overline{\mathbf{x}} \pm \sigma x)\end{array}$ & Parameter & $\begin{array}{c}\text { JT-VMAT } \\
(\overline{\mathbf{x}} \pm \sigma x) \\
\end{array}$ & $\begin{array}{c}\text { SJ-VMAT } \\
(\overline{\mathbf{x}} \pm \sigma x) \\
\end{array}$ & Difference (\%) & p-Value \\
\hline Body & V5 (\%) & $51.608 \pm 8.358$ & $52.086 \pm 8.457$ & 0.926 & 0.009 \\
\hline \multirow{4}{*}{$28777.915 \pm 7364.678$} & $\mathrm{~V} 10(\%)$ & $43.529 \pm 7.338$ & $44.046 \pm 7.451$ & 1.188 & 0.011 \\
\hline & V20 (\%) & $24.744 \pm 3.878$ & $25.093 \pm 3.878$ & 1.410 & $<0.001$ \\
\hline & V30 (\%) & $13.537 \pm 2.460$ & $13.730 \pm 2.587$ & 1.426 & 0.047 \\
\hline & Mean (Gy) & $12.368 \pm 1.875$ & $12.516 \pm 1.881$ & 1.197 & $<0.001$ \\
\hline Bladder & V5 (\%) & $100.000 \pm 0.000$ & $100.000 \pm 0.000$ & 0.000 & - \\
\hline \multirow{4}{*}{$313.328 \pm 92.421$} & $\mathrm{~V} 10(\%)$ & $100.000 \pm 0.000$ & $100.000 \pm 0.000$ & 0.000 & - \\
\hline & $\mathrm{V} 20(\%)$ & $96.753 \pm 10.045$ & $96.836 \pm 9.984$ & 0.086 & 0.248 \\
\hline & V30 (\%) & $81.984 \pm 20.380$ & $82.149 \pm 19.651$ & 0.201 & 0.829 \\
\hline & Mean (Gy) & $38.804 \pm 4.409$ & $30.986 \pm 4.540$ & 0.469 & 0.129 \\
\hline Rectum & V5 (\%) & $100.000 \pm 0.000$ & $100.000 \pm 0.000$ & 0.000 & - \\
\hline \multirow{4}{*}{$69.214 \pm 31.475$} & $\mathrm{~V} 10(\%)$ & $99.911 \pm 0.281$ & $99.971 \pm 0.092$ & 0.060 & 0.343 \\
\hline & V20 (\%) & $98.837 \pm 2.126$ & $98.989 \pm 1.907$ & 0.154 & 0.309 \\
\hline & V30 (\%) & $84.633 \pm 23.295$ & $84.655 \pm 23.458$ & 0.026 & 0.939 \\
\hline & Mean (Gy) & $39.115 \pm 5.135$ & $39.232 \pm 5.338$ & 0.298 & 0.461 \\
\hline Right Femur Head & V5 (\%) & $98.592 \pm 3.192$ & $99.738 \pm 0.552$ & 1.162 & 0.212 \\
\hline \multirow{4}{*}{$83.864 \pm 27.033$} & $\mathrm{~V} 10(\%)$ & $91.220 \pm 13.224$ & $92.611 \pm 10.676$ & 1.525 & 0.409 \\
\hline & V20 (\%) & $41.447 \pm 18.983$ & $42.779 \pm 20.262$ & 3.214 & 0.305 \\
\hline & V30 (\%) & $14.635 \pm 8.230$ & $14.671 \pm 8.493$ & 0.246 & 0.926 \\
\hline & Mean (Gy) & $20.352 \pm 3.973$ & $20.734 \pm 3.816$ & 1.877 & 0.069 \\
\hline Left Femur Head & V5 (\%) & $98.645 \pm 3.151$ & $98.726 \pm 3.263$ & 0.082 & 0.645 \\
\hline \multirow{4}{*}{$83.329 \pm 25.280$} & $\mathrm{~V} 10(\%)$ & $90.516 \pm 14.051$ & $94.048 \pm 9.539$ & 3.902 & 0.181 \\
\hline & $\mathrm{V} 20(\%)$ & $45.915 \pm 22.307$ & $46.016 \pm 20.393$ & 0.219 & 0.946 \\
\hline & V30 (\%) & $17.892 \pm 10.067$ & $17.975 \pm 10.104$ & 0.464 & 0.852 \\
\hline & Mean (Gy) & $21.240 \pm 4.363$ & $21.442 \pm 3.889$ & 0.949 & 0.467 \\
\hline Intestine & V5 (\%) & $90.584 \pm 14.125$ & $91.137 \pm 13.554$ & 0.610 & 0.048 \\
\hline \multirow{4}{*}{$1098.571 \pm 417.814$} & V10 (\%) & $83.125 \pm 15.857$ & $83.228 \pm 15.630$ & 0.124 & 0.706 \\
\hline & V20 (\%) & $58.917 \pm 19.687$ & $59.573 \pm 19.377$ & 1.113 & 0.014 \\
\hline & V30 (\%) & $30.145 \pm 21.738$ & $30.319 \pm 21.054$ & 0.578 & 0.690 \\
\hline & Mean (Gy) & $23.271 \pm 6.628$ & $23.376 \pm 6.437$ & 0.449 & 0.246 \\
\hline \multirow[t]{3}{*}{ Cauda } & V5 (\%) & $84.989 \pm 13.066$ & $86.600 \pm 12.272$ & 1.896 & 0.033 \\
\hline & V10 (\%) & $79.610 \pm 13.922$ & $79.978 \pm 13.906$ & 0.462 & 0.028 \\
\hline & V20 (\%) & $71.444 \pm 14.165$ & $71.746 \pm 14.213$ & 0.423 & 0.275 \\
\hline \multirow[t]{3}{*}{$23.464 \pm 9.838$} & V30 (\%) & $60.199 \pm 10.482$ & $61.256 \pm 12.047$ & 1.756 & 0.512 \\
\hline & Mean (Gy) & $27.572 \pm 4.702$ & $27.646 \pm 4.876$ & 0.268 & 0.751 \\
\hline & Max (Gy) & $45.268 \pm 3.904$ & $45.187 \pm 3.931$ & 0.179 & 0.778 \\
\hline
\end{tabular}

Abbreviation: $\left(\bar{x} \pm \sigma_{\bar{x}}\right)$ is Mean \pm Standard deviation, $V_{n}(\%)$ is the percentage volume receiving ' $n$ ' Gy, JT-jaw tracking, SJ- static jaw 


\section{Discussion}

In this study, we assessed the plan quality using the homogeneity and conformity index for the IMRT/VMAT plans with jaw tracking and static jaws and found that the plan quality indices were comparable and there is no significant difference in the plan quality between jaw tracking and static jaw techniques in IMRT/VMAT. Several studies [2,7-9] found that there is no significant difference in the plan quality between the jaw tracking and static jaw in IMRT/VMAT, but significant differences in the OARs sparing were observed using jaw tracking technique.

Several authors have studied the dosimetric advantages of jaw tracking over the static jaw in IMRT and VMAT plans. But most of the studies were done on multiple sites concurrently, which resulted in diluting the individual site enhanced results. Hence, in this study, we would like to focus only on one site (carcinoma of the cervix) to understand the advantage of the jaw tracking in IMRT and VMAT in-depth. Kim et al. [10] studied the clinical assessment of jaw tracking in IMRT for brain tumors, the differences in the mean doses and the maximum doses to the OARs were larger when the OARs and the planning target volume (PTV) were closer.

Joy et al. [7] investigated the dosimetric effect of JT technique added to the existing clinical plan with step and shoot IMRT using pinnacle TPS (Philips Medical Systems, Madison, WI) for thoracic, head and neck and pediatric patients and found the integral dose was reduced by $2 \%$ and suggested that JT should be introduced during optimization itself for better results. In our study, JT-IMRT was able to demonstrate the average normal tissue $\mathrm{V}_{5}$ volume reduction by $5.40 \%$ and the mean dose of the normal tissue by $4.22 \%$ compared to SJ-IMRT. In the JT-VMAT the $\mathrm{V}_{5}$ and mean dose of the normal tissue was reduced only by $0.92 \%$ and $1.19 \%$ respectively. The risk of the secondary malignancies has a direct correlation with the low dose volumes. In our study, there were significant dose reductions in JT-IMRT of the low dose volumes for the normal tissues, which may certainly reduce the possibility of the secondary malignancies. In JTVMAT the low dose volume reduction for the normal tissues was insignificant or negligible.

The average monitor units (MU) followed by the standard deviation $\left(\overline{\mathrm{x}} \pm \sigma_{\overline{\mathrm{x}}}\right)$ for all the patients in JT-IMRT, SJ-IMRT, JT-VMAT and SJ-VMAT were $1615.900 \pm 216.149,1573.600$ $\pm 206.131,482.5 \pm 124.211$ and $479.600 \pm 124.079$ respectively. We found a mean increase of $2.69 \%$ in MU for
JT-IMRT compared to SJ-IMRT and only $0.60 \%$ increase in MU for JT-VMAT compared to SJ-VMAT. Schmidhalter et al. [11] demonstrated the reduction of leaf transmission with jaw tracking in dynamic IMRT for prostate and head \& neck cancers to the normal tissues by $1.8 \%$ and $1.5 \%$ respectively. This study found that the average MU in the dynamic IMRT was increased by $3.1 \%$ and $2.8 \%$ in prostate and head and neck cancers. Karthick et al. [3] investigated the influence of jaw tracking in head and neck cancers in both IMRT and VMAT. In this study, they found that there is an increase in average MU by $4.21 \%$ for JT-IMRT compared to SJ-IMRT, whereas there is no significant difference in MU between JT-VMAT and SJ-VMAT.

In this work, as the rectum and bladder are located adjacent to the target volume, the lower doses (especially 5Gy and 10Gy volumes) were almost covering the entire bladder and rectum. In the rectum, we found a mean reduction of $1.348 \%$ for JTIMRT compared to SJ-IMRT, in JT-VMAT the reduction was only $0.298 \%$ compared to the SJ-VMAT. Significant dose reduction was observed in $\mathrm{V}_{30}$ for rectum by $3.25 \%$ ( $\mathrm{p}<0.001$ ) and bladder $3.22 \%(\mathrm{p}=0.005)$ respectively.

JT-IMRT resulted in statistically significant dose reduction to all the OARs mean dose and low dose volumes. JT-VMAT also resulted in the dose reduction in the low dose volume and the mean dose to the OARs, but the effects were negligible. In this study, JT-VMAT show an overall reduction in the critical structures of rectum, bladder, cauda, etc., compare to SJVMAT, but we also observed that JT-VMAT resulted in the increased dose in the low dose volume of critical structures in certain patients as shown in Figure 5. This indicates that JT has a definite dosimetric advantage in IMRT plans and negligible or no significant advantages in the VMAT plans.

\section{Conclusion}

Jaw tracking plans with IMRT and VMAT resulted in decreased dose to the OARs without compromising target coverage. But significant dose reductions were observed in IMRT jaw tracking plans. JT-VMAT plans show dose reduction to OARs, still the dose reduction was not highly statistically significant. The reason for significant dose reduction in JT-IMRT plans is that IMRT plans have relatively larger monitor units compared to VMAT plans.

\section{References}

[1] Mani KR, Upadhayaya S, Das KJ. Influence of jaw tracking in intensity-modulated and volumetric-modulated arc radiotherapy for head and neck cancers: a dosimetric study. Radiat Oncol J. 2017;35(1):90-100.

[2] Cadman P, McNutt T, Bzdusek K. Validation of physics improvements for IMRT with a commercial treatment planning system. J Appl Clin Med Phys. 2005;6(2):74-86. 
[3] Eifel PJ, Levenback C, Wharton JT, Oswald MJ. Time course and incidence of late complications in patients treated with radiation therapy for FIGO stage IB carcinoma of the uterine cervix. Int J Radiat Oncol Biol Phys. 1995;32(5):1289-1300,

[4] Hasselle MD, Rose BS, Kochanski JD, et al. Clinical Outcomes of Intensity-Modulated Pelvic Radiation Therapy for Carcinoma of the Cervix. Int J Radiat Oncol Biol Phys. 2011;80(5):1436-1445.

[5] Ng J, Shuryak I. Minimizing second cancer risk following radiotherapy: current perspectives. Cancer Manag Res 2014; $7: 1-11$.

[6] International Commission on Radiation Units and Measurements. Prescribing, recording, and reporting photon beam intensitymodulated radiation therapy (IMRT): contents (ICRU Report 83). J ICRU. 2010;10:NP.

[7] Joy S, Starkschall G, Kry S, et al., Dosimetric effects of jaw tracking in step-and-shoot intensity-modulated radiation therapy. J Appl Clin Med Phys. 2012;13(2):3707.

[8] Feng Z, Wu H, Zhang Y, et al. Dosimetric comparison between jaw tracking and static jaw techniques in intensity-modulated radiotherapy. Radiat Oncol. 2015;10:28.

[9] Park BD, Cho BC, Kim JH, et al. Dosimetric Impact of the Jaw-Tracking Technique in Volumetric Modulated Arc Therapy. J Nucl Med Radiat Ther. 2016; 7:301.

[10] Kim JY, Kim SW, Choe BY, et al. Clinical assessment of the jaw-tracking function in IMRT for a brain tumor. Journal Korean Phys Soc. 2015;66(2):295-300.

[11] Schmidhalter D, Fix MK, Niederer P, et al. Leaf transmission reduction using moving jaws for dynamic MLC IMRT. Med Phys. 2007;34(9):3674-3687. 